\title{
Intimate partner violence screening barriers as perceived by Jordanian nurses: A qualitative descriptive study
}

\author{
Ahlam Al-Natour ${ }^{* 1}$, Abeer Qandil ${ }^{1}$, Gordon Lee Gillespie ${ }^{2}$ \\ ${ }^{1}$ Community and Mental Health Department, College of Nursing, Jordan University of Science and Technology, Irbid, Jordan \\ ${ }^{2}$ College of Nursing, University of Cincinnati, Cincinnati, United States
}

Received: January 19, 2015

Accepted: May 24, 2015

Online Published: June 3, 2015

DOI: $10.5430 /$ jnep.v5n9p11

URL: http://dx.doi.org/10.5430/jnep.v5n9p11

\begin{abstract}
Intimate partner violence screening is an important component of patient assessment; however, not all nurses routinely conduct this screening with their patients. The purpose of this qualitative descriptive study was to describe the barriers to intimate partner violence screening and strategies to overcome these barriers. A purposive sample of 12 male and female Jordanian nurses working at a university hospital in the city of Irbid, Jordan participated in this study. Participants were interviewed using a semi-structured interview guide. Interviews were audio-recorded and transcribed verbatim. Steps of Colaizzi's steps for procedural analysis were used to analyze the transcript data. Four themes were derived from the data: Jordanian culture, system-based IPV screening barriers, IPV victims as barriers to IPV screening, and nurses' attitudes and beliefs for IPV screening. Jordanian nurses encounter many barriers that affect their ability to screen for IPV. Screening for IPV remains an important nursing function even in Jordanian healthcare settings.
\end{abstract}

Key Words: Battered women, Domestic violence, Partner abuse, Qualitative research, Violence

\section{INTRODUCTION}

Globally, it is estimated that up to $75 \%$ of women have undergone physical and sexual intimate partner violence (IPV). ${ }^{[1]}$ In Jordan, the rate of physical IPV has been about $19.6 \%$ and the rate of emotional IPV has been $47.5 \%$ among Jordanian women. ${ }^{[2]}$ Mental and physical health problems including depression, posttraumatic stress disorder, back pain, chronic neck pain, migraines, and digestive problems can result from IPV ${ }^{[3]}$

Although IPV has negatively impacted women's health, nurses IPV screening rates have still been between $10 \%$ and $39 \% .{ }^{[4,5]}$ Al-Natour, Gillespie, Felblinger, and Wang have also found low screening rates among Jordanian nurses and that screening varied by women's chief complaint: $25 \%$ for women seeking care for physical injuries, $20 \%$ for depression and anxiety, $17.8 \%$ for chronic pelvic pain, $14.9 \%$ for hypertension and coronary artery disease, $11.5 \%$ for headaches, and 3.3\% for Irritable Bowel Syndrome. ${ }^{6]}$ An area commonly perceived as most suitable for IPV screening is obstetrics and gynecology; however, only $10.8 \%$ of nurses in these settings screened for IPV.

When detected early, IPV screening can be beneficial for the health of IPV victims. Liebschutz, Battaglia, Finley, and Averbuch indicated that health care providers who inquired and asked about IPV experience were described by their patients as beneficial and helpful. ${ }^{[7]}$ Health care providers

\footnotetext{
*Correspondence: Ahlam Al-Natour; Email: asalnatour@just.edu.jo; Address: College of Nursing, Jordan University of Science and Technology, Irbid, Jordan.
}

Published by Sciedu Press 
reported benefits for victims disclosing their IPV: improved self-esteem, became aware of solutions and alternatives, empowered to seek help and take action, looked for immediate change, assured victims safety after assault, and received emotional support. Furthermore, health care providers screening and supportive role resulted in positive attitudes toward health care providers, and enabled victims to make a safe decision regarding staying with or leaving their abusers. Conversely, victims of IPV reported frustration, disappointment, and anger when their IPV was recognized by health care providers, but not addressed. This lack of health care provider action led to future avoidance of health care. ${ }^{\text {[7] }}$

Nurses are an important professional group concerned with women's health and have close interpersonal contact with women in community health settings. Screening for IPV is a crtical nursing function that can be done at first contact with women in community health settings. Guruge has shown that nurses perceived their roles toward IPV as identifying victims of IPV, providing help and care, assuring victims' safety, referring victims to needed services, providing support, advising victims on their unique circumstances, and raising community awareness to IPV. ${ }^{[8]}$

There appears to be limited consideration of IPV screening among Jordanian nurses and we suspect there may be that barriers to IPV screening that influence the practice as well as the study of IPV screening. Defining nurses' barriers and source of barriers to screening for IPV will help nursing educators, hospital administrators, and health policymakers to overcome IPV screening barriers through nursing education, support, and legislation for universal screening. Helping nurses define the suffering of patients and control the barriers to IPV screening may improve their nursing practice. Therefore the purpose of this study was to describe the personal, cultural, and institutional barriers for Jordanian nurses to screen for IPV and strategies to overcome these barriers. The future implementation of derived strategies recommended by the sample may lead to greater detection of IPV cases early in their course and decrease the hazardous impact of IPV against Jordanian women accordingly.

\section{MethodS}

This study used a qualitative descriptive design with a conventional content analysis approach. Research using a qualitative descriptive design will "offer a comprehensive summary of an event in the everyday terms of those events" (p. 336). ${ }^{[9]}$ Generating this comprehensive summary can be accomplished using a conventional content analysis approach, because this approach "is generally used with a study design whose aim is to describe a phenomenon" (p. 1279) that is multi-faceted and sensitive such as IPV. ${ }^{[10]}$

\subsection{Sampling}

Purposive sampling was employed in this study. Purposive sampling helps enrich the data to yield transferable findings, because purposive samples provide detailed responses relevant to a study purpose. ${ }^{[11]}$ Twelve nurses ( 6 males and 6 females) working at a single university hospital were asked to participate in this study. Inclusion criteria for this study were being Jordanian, over the age of 22, and responsible for communicating with female patients and addressing IPV.

\subsection{Ethical considerations}

Each participant received an information sheet that included the study purpose, participation benefits and risks, and their right to withdraw from the study at any time. Participants were encouraged to ask questions. Privacy was maintained during the interview and participants gave permission to record the interviews. Confidentiality was maintained by not using any participant names during the interviews or documenting their names on the transcript data.

\subsection{Data collection process}

After approval by the IRB, the first author approached hospital administrators and head nurses to discuss participant recruitment. Female nurses working in female wards and male nurses working in the emergency department were approached to participate. Participants were individually interviewed at a time and setting convenient for each participant. Data were collected through face-to-face semi-structured interviews. Interviews were audio recorded. Field notes were used to document participants' nonverbal communication during the interviews.

During the interview, participants were asked to describe their personal detailed experience with barriers for IPV screening. Participants responded to the following openended questions:

(1) What are the barriers that you encounter when caring for women undergoing IPV?

(2) How can nurses overcome IPV screening barriers?

Interviews lasted about 30-45 minutes. Audio recordings were transcribed verbatim. Data collection was terminated when data saturation was reached and no new themes emerged.

\subsection{Data analysis}

The data were analyzed using Colaizzi's seven steps of procedural analysis. ${ }^{[12]}$ First the transcripts were read and reread by the first two authors to garner a sense of the barriers to IPV screening as reported by the participants. Second, significant statements pertaining to barriers of IPV screening were extracted independently by these two authors. Third, 
these two authors independently formulated meanings for the statements and then discussed each statement/meaning until they came to agreement. Fourth, these two authors categorized the meanings into clusters of themes. The third author then reviewed the findings to determine their credibility in relation to the themes derived from the data. Fifth, all three authors integrated the findings into an exhaustive description of barriers to IPV screening. Sixth, the findings were validated with the 12 research participants. The final step, step 7, was not needed, because the participants agreed with the findings and did not offer any changes.

\subsection{Trustworthiness}

Transferability, credibility, and confirmability were used to increase trustworthiness and assure study rigor. ${ }^{[13]}$ Transferability demonstrates the application of the findings from this study to the larger study population. Transferability was achieved in this study by the rich and thick description and with relevant exemplars. Also a detailed information and description of their actual situation and experiences by study informants and their cultural context assured the transferability of findings.

Credibility is the accurate identification, description, and reflection of the study phenomenon. Credibility was assured in this study through member checking of transcripts and verification of the findings with the study sample. Interviews with informants and repeating questions were used to assure credibility. Informants were specifically asked to clarify or add to the description of the findings.

Confirmability is similar to the concept of objectivity in quantitative research designs. In this study, confirmability was achieved through bracketing. Bracketing was assured when the investigator sets aside preconceived notions and assumptions about a phenomenon prior to data collection and analysis. This allowed the researchers to position themselves to see the phenomenon through the eyes of the study participants. To further assure confirmability, a third author reviewed the study findings to confirm that the statements coded to each theme were relevant and the description of that theme represented the data coded to it.

\section{RESUlts}

Twelve Jordanian nurses (6 males, 6 females) participated in this study. Their mean age was 29 years. Their mean years of nursing experience were 7 years. All of the nurses were Jordanian Muslims. Ten participants attained a bachelor degree and two a master's degree. Six participants worked in the emergency department, two in the labor and delivery department, two in the maternity department, and two in the maternal surgery department. Eleven participants were married and one was single. All participants lived in Irbid, Jordan.

Four themes were derived from the semi-structured interviews with Jordanian nurses that reflected barriers to IVP screening: (1) Jordanian culture, (2) system-based IPV screening barriers, (3) IPV victims as barriers to IPV screening, and (4) nurses' attitudes and beliefs for IPV screening.

\section{Theme 1: Jordanian culture}

The first type of IPV screening barrier was the Jordanian culture itself. Jordanian nurses described the conservative Jordanian Arabic Muslim cultural traditions and beliefs as significant barriers. Jordanian culture also was described as male-dominated giving men authority, privileges, and rights over women. This male domination was demonstrated by perpetrators repeating the IPV they witnessed as children. Also, women related to these cultural traditions should accept violent and stressful events in their lives for the sake of their children, home, and their family of origin reputation as well as the IPV being a private familial issue. A relevant exemplar was "Our Jordanian traditions indicate the dominant role of male over female, men's rights to abuse women. Women could not disclose violence for the sake of herself and her family, and the absence of family support." Male nurses indicated that victims disclosing IPV could result in destroying the family. Related to this cultural norm, women would not be allowed to disclose violence. For example, a male nurse said, "In our community, seeking court and legal action is irreversible way for women, which means the end of family life for both."

\section{Theme 2: System-based IPV screening barriers}

The second type of IPV screening barrier was system-based IPV screening barriers. These barriers were described in terms of lack of time to screen, priority of nursing care, absence of privacy to screen, absence of educational and training programs for screening, screening was not required, and inactive role of social and mental specialist. Nurses indicated lack of time was a big constraint for screening which when done resulted in work overload and was further complicated by a shortage of nursing staff. Nurses indicated their priority of care for medical care over screening by a male nurse's response, "When I am working in the emergency department, I am giving priority to patients with injuries and trauma, and severe cases. It is better than asking and screening victims for IPV." An absence of privacy for screening was reflected in the statement by a male nurse: "In this hospital there is no private special place for screening. There is a need for quiet, private places which facilitate communication and promote disclosing." The absence of educational and training 
programs was seen in the responses by 11 participants including the following exemplar: "I got an education session about domestic violence for two hours two years ago, and it was very brief and non-beneficial." Screening not being required was verified by the statement, "In our institution, we lack protocols for screening for IPV, and lack a policy for indicating the nurse's role in screening." The lack of policy was further complicated by the lack of a valid feasible tool for IPV screening. Some nurses indicated the absence of mental or social services at this hospital. When the services were available, mental and social service specialists did not have a role or active role in helping or intervening for IPV. For example, a male nurse from the emergency department said, "I know that there is a social worker at the hospital, but he is unaware of his role." Some participants communicated that if the organization was more supportive, more safety measures were available, and nurses were authorized to manage women identified as victims of IPV that IPV screening would more likely occur.

\section{Theme 3: IPV victims as barriers to IPV screening}

The third type of IPV screening barrier was the victims themselves. Jordanian nurses described victims as a barrier, because victims were afraid of perpetrators' retaliation, losing custody of children, getting divorced, and sometimes victims are at least partially responsible for the abuse. Nurses said, "Nurses accept violence because of the stigma associated with divorce, losing children, perpetrators retaliation after disclosing to health provider." Also nurses indicated that victims' had a lower self-esteem and confidence and unawareness of their rights could result in women refusing to disclose or seek help. One male nurse explained that victims' low self-esteem and confidence resulted in violence against women by their husbands. For example, "Women's low selfesteem and confidence makes victims accept violence in their lives." Some Jordanian nurses believed victims were at least partially responsible for the abuse encountered. For example, two female nurses and two male nurses conveyed a sentiment similar to this one: "Mostly victims are abused because they are not obeying their husband's commands, or she is misbehaving with her in-laws." These perceptions of women who were victimized could have been a projection of some participants' experience with IPV. For example, one female nurse expressed her experience as "My husband abused me verbally, and I am angry with that. I have that bad experience; I am unable to help myself. How can I help other victims?"

Theme 4: Nurses' attitudes and beliefs for IPV screening The fourth type of screening barrier was nurses' attitudes and beliefs. Primary barriers related to nurses' emotions (e.g., fear, anger, frustration, guilt). Fear was detailed as fear for victims' safety, personal safety, offending victims, and nurses losing their jobs. Jordanian nurses expressed their fears toward victims' safety if they disclosed their experience of IPV to nurses or to health care providers. An example of fear for a victim's safety by a female participant was "I am afraid that her husband will retaliate and seek revenge for her disclosing violence, especially if she has no family support." Anger was expressed by participants, because of the abuse women suffered and the extreme empathy they experienced for victimized women. Jordanian nurses were frustrated by not being able to provide help or proper intervention for victims of marital violence or not being knowledgeable of their roles and responsibilities for victimized women. Additional barriers to IPV screening related to nurses' attitudes and beliefs were a lack of awareness or denial of their role and responsibility, perceived inability to help, belief that perpetrators may be suffering from mental health problems, victims will not disclose to male nurses, and community could not provide support or help for women. Of particular note was the perceived lack of community support if an IPV victim was identified. Nurses who considered screening were challenged by the belief that no community resources were available to victims. One female nurse said, "I cannot help victims or refer them to services that can provide help, also our culture could not provide help or support for victimized women."

\section{Discussion}

Several types of barriers to IPV screening were identified in this study: Jordanian culture, system-based IPV screening barriers, IPV victims, and nurses' attitudes and beliefs. The conservative Jordanian culture limited the screening of IPV. An initial step to changing the culture of secrecy in Jordan relative to IPV may be increasing awareness about the rights of married women in Jordan within Islamic doctrine and Jordanian law. Educating women and the community about their legal rights can benefit women experiencing IPV. ${ }^{[14,15]}$ Equally important, community education may lead to nurses emphasizing the importance for increasing and promoting community awareness.

Given the perceived system-based barriers against IPV screening at the study site, interventions are warranted that promote and permit IPV screening. Al-Natour et al., ${ }^{[6]}$ Baig et al., ${ }^{[15]}$ Felblinger and Gates, ${ }^{[16]}$ Furniss et al., ${ }^{[17]}$ and Haggblom and Moller ${ }^{[14]}$ recommended the need for more contact time between nurses and patients. Not having sufficient contact time with patients, limited some nurses from screening for IPV. Time is an important factor for IPV. While more direct time is needed to conduct IPV screening, Jordanian nurses were already overloaded with multiple nursing tasks 
which they assigned a clinical priority. Time as a barrier when direct clinical care also was needed was reflected in the findings of several other studies. ${ }^{[8,15,18]}$ This lack of time adversely affects nurses' ability to build trusting relationships needed to address IPV. ${ }^{[8]}$ Offering social and mental services to victims and establishing private and safe places to screen, and establishing and enforcing screening guidelines can address many of the participants' concerns that prevented them from screening. While mental and social services are important for IPV care, many Jordanian nurses do not value or understand this role for helping victims of IPV. ${ }^{6]}$

Factors related to IPV victims were exemplified as barriers to IPV screening. Specifically, the nurse participants in this study believed that victims were at least partially responsible for their abuse. In other research, some nurses believed that women chose to be victims of IPV. ${ }^{[6]}$ While the belief of these nurses may be arguable, it is important to note that this belief exists. Even when women choose to remain in violent relationships, nurses need to help victims identify the reasons why remain. For example, nurses in the current study discussed that IPV victims were afraid of retaliation from the perpetrators, becoming divorced, and losing custody of their children. Despite these perceived barriers, nurses need to consider screening for IPV and helping victims develop safety plans when returning to violent relationships so that the physical and mental harm from these relationships can be mitigated or reduced.

Nurses' attitudes and beliefs as a barrier to IVP screening identified in this study were consistent with findings from other studies. Haggblom and Moller ${ }^{[14]}$ and Todahl, Linville, Chou, and Maher-Cosenza ${ }^{[19]}$ indicated that nurses were concerned about victims' safety because perpetrators violence often escalated after the IPV was reported. A similar concern for victims' safety was identified in the current study. Therefore, nurses may opt to not screen for IPV in an effort to prevent further harm to their patients. However, findings in the current study with Jordanian nurses were contrary to findings from Furniss et al.'s study which was conducted with nurses who screen for IPV in the United States. ${ }^{[17]}$ Nurses in Furniss et al.'s study were not aware of the risk to their personal safety should they screen for IPV in the presence of the offender. ${ }^{[17]}$ In the current study, nurses were quite aware of this risk which was identified a key barrier that prevented some of the nurses from screening for IPV. This perceived risk was exemplified as a fear of perpetrators, because the perpetrators could become violent and cause physical or mental harm to nurses. Respondents in studies by Al-Natour et $a l^{[6]}$ and Guruge ${ }^{[8]}$ with nurses from Jordan and Sri Lanka respectively reported similar concerns for personal safety and being afraid of perpetrators' potentially violent responses.

Published by Sciedu Press
Not all nurses believe they are responsible to screen for IPV. ${ }^{[6,16,17]}$ This belief also was found in the current study. A potential reason for this finding could be a belief that victims are responsible for the violence against them. In a study of Jordanian nurses, Al-Natour et al. reported that more than half of their participants believed that victims are responsible for IPV. ${ }^{[6]}$ In the Jordanian culture, women are expected to obey and follow husbands' "commands" and are "legitimately" victimized when they do not comply. ${ }^{[6,20]}$ Another potential reason for the blaming of victims is the passive personality of victims who "choose" to stay in abusive relationship or not seek help. ${ }^{[6]}$ Khawaja, Linos, and El-Roueiheb found that $60 \%$ of Jordanian women and men justify and rationalize IPV as a way to control women. ${ }^{[21]}$

Nurses' personal victimization was identified as another influencer for IPV screening while some nurses may become more empathetic IPV screeners. Minsky-Kelly, Hamberger, Pape, and Wolff stated that being a victim of IPV could hinder nurses' in screening and prevent them from intervening on behalf of victims of IPV. ${ }^{[22]}$ Todahl, Linville, Chou , and Maher-Cosenza posited that nurses who experienced IPV increased their awareness when screening for IPV. ${ }^{[19]}$ While there is legitimacy in the beliefs of the participants of the current study who agreed with Todahl et al.'s position, ${ }^{[19]}$ personal victimization was not assessed. Further research is needed to explore the relationships of empathy, victimization, screening, and referral. Given the high rate of victimization found by Al-Natour et al. against female Jordanian nurses, this could be particularly problematic with future efforts to increase IPV screening. ${ }^{[23]}$

This study may be limited by measurement bias given that researchers themselves are instruments in qualitative research. This limitation was minimized by having the same researcher conduct all the participant interviews such that the effect, if any, would have been the same for each study participant.

\section{Conclusion}

Jordanian nurses encounter many barriers that affect their ability to screen for IPV. Screening for IPV remains an important nursing function even in Jordanian healthcare settings. Nurses in collaboration with hospital administrators can develop strategies that address nurses' attitudes, nurses' beliefs, and institutional barriers preventing nurses from screening for IPV. Strategies need to be culturally congruent with the belief systems of Jordanian women. Further research is needed to evaluate the efficacy of any strategies implemented to increase IPV screening.

\section{CONFLicts OF INTEREST Disclosure}

The authors declare that there is no conflict of interest statement. 


\section{REFERENCES}

[1] Garcia-Moreno C, Jansen HA, Ellsberg M, et al. Prevalence of intimate partner violence: Findings from the WHO multi country study on women's health and domestic violence. Lancet. 2006; 368: 1260-1269. http://dx.doi.org/10.1016/S0140-6 $736(06) 69523-8$

[2] Al-Nsour M, Khawaja M, Al-Kayyali G. Domestic violence against women in Jordan: Evidence from health clinics. J Family Violence. 2009; 24: 569-575. http://dx.doi.org/10.1007/s10896-009 $-9255-2$

[3] Campbell JC. Health consequences of intimate partner violence. Lancet. 2002; 359: 1331-1336. http://dx.doi.org/10.1016/S 0140-6736(02) 08336-8

[4] Malecha A. Screening for and treating intimate partner violence in the workplace. AAOHN J. 2003; 51: 310-316. PMid:12880235

[5] Thurston WE, Tutty LM, Eisener AE, et al. Domestic violence screening rates in a community health center urgent care clinic. Research Nurs Health. 2007; 30: 611-619. PMid:18022814 http: //dx.doi.org/10.1002/nur. 20221

[6] Al-Natour A, Gillespie GL, Felblinger D, et al. Jordanian nurses barriers to screening for intimate partner violence. Violence Against Women. 2014; 20: 1473-1488. PMid:25421285 http://dx.doi.o rg/10.1177/1077801214559057

[7] Liebschutz J, Battaglia T, Finley E, et al. Disclosing intimate partner violence to health care clinicians - What a difference the setting makes: A qualitative study. BMC Public Health. 2008; 8 : 229. PMid:18601725 http://dx.doi.org/10.1186/1471-245 8-8-229

[8] Guruge S. Nurses' role in caring for women experiencing intimate partner violence in the Sri Lankan context. Int Scholarly Research Network. 2012; 1-8.

[9] Sandelowski M. Whatever happened to qualitative description? Res Nurs Health. 2000; 23: 334-340. http://dx.doi.org/10.1002 /1098-240X (200008) $23: 4<334:$ : AID-NUR9>3 . 0. CO ; $2-G$

[10] Hsieh H, Shannon SE. Three approaches to qualitative content analysis. Qualitative Health Res. 2005; 15(9): 1277-1288. PMid:16204405 http://dx.doi.org/10.1177/1049732305276687

[11] Jupp V. Purposive sampling. In The SAGE Dictionary of Social Research Methods (pp. 245-246). London, England: SAGE Publications; 2006.

[12] Colaizzi P. Psychological research as the phenomenologist views it In Valle R, King M (Eds). Existential-phenomenological Alternatives for Psychology. New York, NY: Oxford University Press; 1978
[13] Lincoln YS, Guba EG. Naturalistic inquiry. Newbury Park, CA: SAGE Publications; 1985.

[14] Haggblom AME, Moller AR. On a life-saving mission: Nurses' willingness to encounter with intimate partner abuse. Qualitative Health Research. 2006; 16: 1075-1090. PMid:16954526 http: //dx.doi.org/10.1177/1049732306292086

[15] Baig AA, Ryan GW, Rodriguez MA. Provider barriers and facilitators to screening for intimate partner violence in Bogota, Colombia. Health Care Women International. 2012; 33: 250261. PMid:22325025 http://dx.doi.org/10.1080/07399332. 2011.646368

[16] Felblinger DMR, Gates D. Domestic violence screening and treatment in the workplace. AAOHN J. 2008; 56: 143-150. http: //dx.doi.org/10.3928/08910162-20080401-04

[17] Furniss K, McCaffrey M, Parnell V, et al. Nurses and barriers to screening for intimate partner violence. Am J Maternal Child Nurs. 2007; 32: 238-243. PMid:17667289 http://dx.doi.org/10.10 97/01. NMC. 0000281964.45905 .89

[18] Denise MD, Kennedy C, Knapp JF, et al. Health care providers' perspectives on screening for intimate partner violence in a pediatric emergency department. Archives Pediatric Adolescent Med. 2002; 156: 794-799. http://dx.doi.org/10.1001/archpedi.156.8. 794

[19] Todahl JL, Linville D, Chou LY, et al. A qualitative study of intimate partner violence universal screening by family therapy interns: Implications for practice, research, training, and supervision. J Marital Family Therapy. 2008; 34: 28-42.

[20] Oweis A, Gharaibeh M, Al-Natour A, et al. Violence against women: Unveiling the suffering of women with a low income in Jordan. J Transcultural Nurs. 2009; 20: 69-76. PMid:18832763 http://dx.doi.org/10.1177/1043659608325848

[21] Khawaja M, Linos N, El-Roueiheb Z. Attitudes of men and women towards wife beating: Findings from Palestinian refugee camps in Jordan. J Family Violence. 2008; 23: 211-218. http: //dx.doi .o $\mathrm{rg} / 10.1007 / \mathrm{s} 10896-007-9146-3$

[22] Minsky-Kelly D, Hamberger LK, Pape DA, et al. We've had training, now what? Qualitative analysis of barriers to domestic violence screening and referral in a health care setting. J Interpersonal Violence. 2005; 20: 1288-1309. PMid:16162490 http://dx .doi .org /10.1177/0886260505278861

[23] Al-Natour A, Gillespie GL, Wang LL, et al. A comparison of intimate partner violence between Jordanian nurses and Jordanian women. J Forensic Nurs. 2014; 10: 13-19. PMid:24434945 http: //dx.doi.org/10.1097/JFN. 0000000000000016 